\title{
Effect of glucocorticoids on osteoclast function in a mouse model of bone necrosis
}

\author{
MING HE，JIASHI WANG，GUANGBIN WANG，YE TIAN，LINLIN JIANG, \\ ZHAOZHOU REN, CHUANG QIU and QIN FU
}

Department of Orthopedics, Shengjing Hospital of China Medical University, Shenyang, Liaoning 110004, P.R. China

Received May 18, 2015; Accepted February 25, 2016

DOI: $10.3892 / \mathrm{mmr} .2016 .5368$

\begin{abstract}
Osteonecrosis, also termed aseptic necrosis, is the cellular death of bone components due to interruption of the blood supply. Glucocorticoid (GC) therapy is a common non-traumatic cause of osteonecrosis. However, the mechanism by which GCs induce osteonecrosis remains to be elucidated. The aim of the present study was to investigate the effects of GCs on osteoclast and osteoblast differentiation and function in a $\mathrm{GC}$-induced osteonecrosis mouse model. BALB/c male mice ( $\mathrm{n}=40 ; 4$-weeks-old) were treated with dexamethasone and asparaginase for 8 weeks. The control group $(n=20)$ was administered normal saline. The results demonstrated that the GC-treated group had a lower mean weight compared with the control group. Morphologically, 16/37 (43\%) mice demonstrated significant osteonecrotic lesions in the GC-treated group. However, osteonecrotic lesions were not observed in the mice of the control group. Furthermore, immunohistochemistry demonstrated that the GC-treated group had a higher level of osteoprotegerin compared with the control group, without any change in the expression of receptor activator of nuclear factor- $\kappa \mathrm{B}$ ligand. In addition, tartarate-resistant acid-phosphatase staining demonstrated significantly decreased osteoclasts in the areas of bone destruction in the GCs-treated group. Furthermore, the present study demonstrated that GCs increased expression levels of osterix and osteocalcin, and decreased expression of matrix metallopeptidase- 9 to regulate the differentiation and function of osteoblasts and osteoclasts. The results of the present study suggested that GCs influence bone remolding resulting in decreased osteoclasts formation/differentiation. Therefore, regulating the differentiation and activity of the osteoclasts may be beneficial to the control and treatment of osteonecrosis.
\end{abstract}

Correspondence to: Dr Qin Fu, Department of Orthopedics, Shengjing Hospital of China Medical University, 36 Sanhao Street, Shenyang, Liaoning 110004, P.R. China

E-mail: topheming56@163.com

Key words: glucocorticoid, osteonecrosis, osteoclast, osteoblast

\section{Introduction}

Osteonecrosis is a disease caused by reduced blood flow to the joints, leading to bone necrosis, and gradually disability, despite treatment. In healthy individuals, the bone breaks down and rebuilds into a new bone, while the old bone is resorbed and replaced. The process maintains the skeleton strong and aids in maintaining a balance of minerals. However, in the process of osteonecrosis, the healing process is usually ineffective, as the bone tissue breaks down faster than the body's ability to repair it. If left untreated, the disease progresses, the bone collapses (1) and the joint surface breaks down, leading to pain and arthritis.

Although osteonecrosis is demonstrated in numerous parts of the human skeleton, the femoral head is the most commonly affected bone. The incidence rate of osteonecrosis is increasing worldwide and further research is being performed. However, the pathogenesis of the disease remains unclear despite decades of research. Various theories have been proposed to analyze the pathophysiological mechanisms of the disease, including fat embolism in the microcirculation or intravascular coagulation, and intraosseous hypertension (2). These theories are not only mutually exclusive, but support each other in various cases (3). The proposed risk factors include chemotherapy, alcoholism (4), excessive steroid use (5), post-trauma $(6,7)$, caisson disease (2), vascular compression (8), hypertension, vasculitis, arterial embolism and thrombosis, damage from radiation, bisphosphonates (9), sickle cell anemia (10) and Gaucher's Disease.

Glucocorticoid (GC) therapy is a non-traumatic cause of osteonecrosis. The prevalent hypothesis is that hypertrophy of bone-marrow adipocytes leads to an increase in bone-marrow pressure, resulting in reduced blood flow in the sinusoids and ischemia. GCs may have a direct toxic effect on bone cells and serve an important role in bone cell apoptosis. This hypothesis may explain the incidence of osteonecrosis in patients with diseases that require long-term glucocorticoid treatment, including leukemia or connective tissue diseases (11). In the process of bone necrosis, the effect of GCs on bone remolding or GC treatment on osteoclasts and osteoblasts, and the mechanisms underlying this remains to be elucidated.

The aim of the present study was to investigate the effects of GCs on osteoclast and osteoblast differentiation, and function in a GCs-induced osteonecrosis mice model. 
Dexamethasone and asparaginase were utilized for treatments, as they are widely used in the clinical treatment of leukemia. BALB/c male mice $(n=40 ; 4$-weeks-old $)$ were treated with $0.4 \mathrm{mg} / \mathrm{kg} /$ day dexamethasone and 7,500 IU/kg/week asparaginase for 8 weeks. At the same time, the control group $(n=20)$ was treated with normal saline through identical administration. The weight of the mice was determined and compared between the GCs-treated and the control groups. The morphological changes induced by dexamethasone and asparaginase were observed using hematoxylin and eosin (H\&E) staining. Furthermore, alterations in the expression levels of the receptor activator of nuclear factor $-\kappa \mathrm{B}$ ligand (RANKL) and osteoprotegerin (OPG) were determined with immunohistochemistry, and the tartarate-resistant acid-phosphatase (TRAP) staining was utilized to identify osteoclasts. Finally, osterix, Runx2, osteocalcin and matrix metallopeptidase (MMP)-9 are associated with differentiation and the activity of osteoclasts and osteoblasts. Therefore, their mRNA expression levels were assessed with quantitative polymerase chain reaction (qPCR). The results demonstrated that dexamethasone and asparaginase influence the bone remolding and result in decreased osteoclasts formation/differentiation. The data suggested that GCs reduce the ability of osteoclasts to reabsorb the 'old bone' to a certain extent. Therefore, regulating the differentiation and activity of the osteoclasts may be beneficial to the control and treatment of osteonecrosis.

\section{Materials and methods}

Reagents. Dexamethasone injection was purchased from Runzhong Pharmaceutical Co., Ltd. (Henan, China), and asparaginase injection from Changzhou Benny Biochemical Pharmaceutical Co., Ltd. (Changzhou, China). Tetracycline hydrochloride injection was purchased from New Asia Pharmaceutical Co., Ltd. (Shanghai, China), and sulfamethoxazole injection from Fang Ming Pharmaceutical Group Co., Ltd. (Shandong, China). Sodium chloride (0.9\%) injection was purchased from Huaren Pharmaceutical Co., Ltd. (Qingdao, China). Low-folate diet and standard rodent diet were purchased from Beijing HFK Bioscience Co., Ltd. (Beijing, CHN). A TRAP/alkaline phosphatase (ALP) staining kit (code No. 294-67001) was obtained from Wako Pure Chemical Industries, Ltd. (Wako, Japan). RANKL (bs-0747R) and OPG (bs-0431R) antibodies were obtained from Beijing Biosynthesis Biotechnology Co., Ltd. (Bioss, China).

Animals and diet. BALB/c male mice (30 g; $\mathrm{n}=60$; 4-weeks-old; Beijing HFK Bioscience Co., Ltd) were tagged and bred in plastic cages (5 mice/cage) under standard laboratory conditions with a $12 \mathrm{~h} \mathrm{light/dark} \mathrm{cycles,} \mathrm{at} \mathrm{a}$ constant temperature of $20^{\circ} \mathrm{C}$ and humidity of $48 \%$. Food and water were provided ad libitum. The mice received a low-folate diet or a standard rodent diet, according to the experimental design. The mice were weighted daily until the end of the experiment. All experiments were performed under an animal protocol approved by the Laboratory Animal Committee of the China Medical University. After 8 weeks, mice from both groups were sacrificed under isoflurane anesthesia (Shanghai Xin Yu Biotech Co., Ltd., Shanghai, China).
GCs and asparaginase administration. Mice were randomly divided into two groups as follows: i) Control, 20 mice treated with saline; ii) GC-treated, 40 mice treated with dexamethasone $(0.4 \mathrm{mg} / \mathrm{kg} / \mathrm{day}$; intragastric) and asparaginase (7,500 IU/kg/week; intraperitoneally) for 8 weeks.

Tissue collection and pre-treatment. The left femur specimens from each group were collected and trimmed of excess tissue, and fixed in $10 \%$ neutral buffer formalin (Shanghai Xin Yu Biotech Co., Ltd.) for $48 \mathrm{~h}$ at room temperature (RT). Bone specimens were decalcified in $10 \%$ ethylenediaminetetraacetic acid solution for 2 weeks at RT. The tissues were subsequently placed in $70 \%$ ethanol and embedded in paraffin.

$H \& E$ staining. Tissue sections $(5 \mu \mathrm{m})$ were obtained and stained using H\&E for routine morphological analysis. Briefly, the sections were deparaffinized in xylene (Shanghai Xin Yu Biotech Co., Ltd.), rehydrated with graded alcohol (Shanghai Xin Yu Biotech Co., Ltd.) and washed three times in distilled water. Subsequent to the washes, hematoxylin (Shanghai Xin Yu Biotech Co., Ltd.) was utilized to stain for nuclei for $2 \mathrm{~min}$. The sections were then rinsed in running tap water and differentiated with $0.3 \%$ acid alcohol (Shanghai Xin $\mathrm{Yu}$ Biotech Co., Ltd.). Following rinsing, the sections were stained with eosin (Shanghai Xin Yu Biotech Co., Ltd.) for 2 min, dehydrated in ethanol and mounted. Osteonecrosis was defined using the following criteria: Empty lacunae, pyknotic nuclei of ghost osteocytes in the bone trabeculae and necrosis of the adjacent marrow (12-14). Mice with $\geq 1$ osteonecrotic lesions were considered positive for osteonecrosis and those with no osteonecrotic lesions were considered negative.

Bone immunohistochemistry for RANKL and OPG expression, and TRAP staining. Bone immunohistochemistry for RANKL and OPG expression were assessed, as previously described (15). Rabbit polyclonal RANKL (1:3,000; cat. no. ab9957; Abcam, Cambridge, MA, USA) and OPG primary antibodies (1:1,00; cat. no. ab183910; Abcam) were used for the staining of paraffin-embedded bone sections. Subsequently, the sections were incubated with peroxidase-coupled goat anti-rabbit secondary antibody (1:200; cat. no. ab97051; Abcam) at $37^{\circ} \mathrm{C}$ for $2 \mathrm{~h}$. Additional bone sections were stained cytochemically for TRAP activity. A TRAP/ALP stain kit was used for TRAP staining of paraffin-embedded bone sections as follows: Section $(4 \mu \mathrm{m})$, drying, staining and mounting. Images of RANKL, OPG and TRAP sections were digitally captured with a Nikon DS-Fil camera (Nikon Corporation, Tokyo, Japan) linked to an Olympus BX-51 microscope (Olympus, Tokyo, Japan) attached to a computer with the NIS-Elements F software, version 4.3 (Nikon Corporation). The expression levels of RANKL and OPG were determined by measuring the optical density and relative area of staining, respectively. The number of TRAP positive multinucleated cells, along with the trabecular bone length, were subsequently enumerated and expressed as osteoclasts per millimeter of trabecular bone length.

$q P C R$. The right femurs were crushed under liquid nitrogen conditions using a Kinematica tissue pulverizer (Shanghai Xin Yu Biotech Co., Ltd.) and RNA was isolated using RNeasy 
Mini kit, according to the manufacturer's protocol (Qiagen, Inc., Valencia, CA, USA). The total RNA concentration was assessed using a NanoDrop 1000 spectrophotometer (Thermo Fisher Scientific, Inc., Waltham, MA, USA). The mRNA expression levels of the genes encoding osterix, osteocalcin, MMP-9 and runt-related transcription factor 2 (Runx2)/core-binding factor $\alpha 1$ (Cbfa1) were measured by qPCR. qPCR was performed using the Stratagene Mx3000P qPCR system (Qiagen, Inc., Valencia, CA, USA), according to the manufacturer's protocol and SYBR Bio Premix Ex Taq (Takara Biotechnology Co., Ltd., Dalian, China) as a DNA-specific fluorescent dye. qPCR was performed for 50 cycles of $95^{\circ} \mathrm{C}$ for $10 \mathrm{sec}$ and $60^{\circ} \mathrm{C}$ for $30 \mathrm{sec}$. Primer sequences were as follows: Osterix, sense: $5^{\prime}$-CGCATCTGA AAGCCCACTTG-3' and antisense 5'-CAGCTCGTCAGA GCGAGTGAA-3'; osteocalcin, sense: 5'-CTTGAAGACCGC CTACAAAC-3' and antisense: 5'-GCTGCTGTGACATCCA TAC-3'; MMP-9, sense: 5'-CCATGCACTGGGCTTAGA TCA-3' and antisense: 5'-GGCCTTGGGTCAGGCTTAGA-3'; Runx2, sense: 5'-TGGACCTTTCCAGACCAGCAGCA-3' and antisense: 5'-GGCAAGTTTGGGTTTAGCAGCGT-3'; GAPDH, sense: 5'-TCGTGCGTGACATTAAGGAG-3' and antisense 5'-ATGCCAGGGTACATGGTGGT-3'. All reactions were repeated a minimum of three times. mRNA expression levels were normalized against the housekeeping gene, GAPDH, using the Stratagene Mx3000P software (Agilent Technologies, Inc., Santa Clara, CA, USA) and fold changes were calculated using the $2^{-\Delta \Delta \mathrm{Cq}}$ normalization method (16).

Statistical Analysis. Data are expressed as the mean \pm standard deviation. Statistical analysis of the data was performed using one-way analysis of variance with the SPSS software, version 11.5 (SPSS, Inc., Chicago, IL, USA). $\mathrm{P}<0.05$ was considered to indicate a statistically significant difference. All experiments were repeated a minimum of three times.

\section{Results}

Effect of GC treatment on mice weight. The survival rate of the GC-treated group (92.5\%; 37/40 mice), prior to mice sacrifice, indicated no significant difference compared with the control group (95\%; 19/20 mice; $\mathrm{P}>0.05$ ). Furthermore, as demonstrated in Fig. 1, the mean weight of mice for the same time period in the GC-treated group $(19.8 \pm 1.9 \mathrm{~g})$ was significantly lower compared with those of mice in the control group $(25.6 \pm 1.5 \mathrm{~g})$ after 8 weeks of treatment $(\mathrm{P}<0.05)$.

Effect of GCs treatment on the histological morphology of bone formation. All mice were sacrificed under isoflurane anesthesia after the 8 week experimental period. The left femur specimens were collected and the histological changes of femur specimens were observed under a light microscope. Osteonecrosis was observed in $16 / 37$ mice $(43 \%)$ in the GC-treated group, and no osteonecrotic lesions were observed in the control group. Fig. 2 shows the histopathological appearance of the femoral head following H\&E staining. Empty lacunae within the necrotic bone trabeculae, bone marrow cells, including adipocytic necrosis, and an accumulation of cell debris in the medullary space in most areas of the femoral

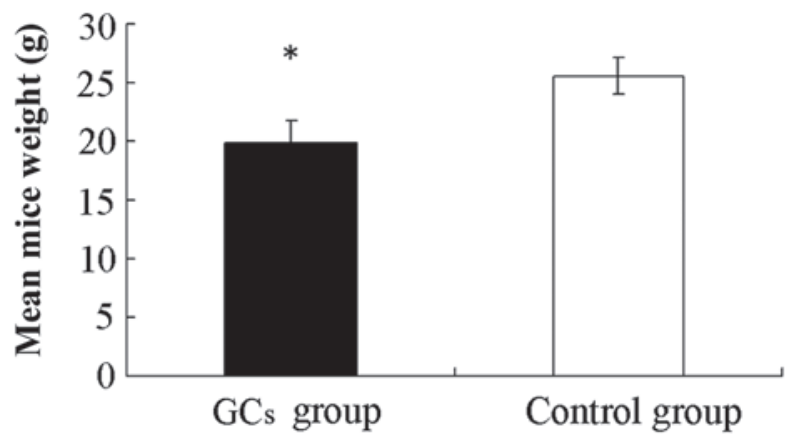

Figure 1. Effects of GCs on mice weight. The mice were sacrificed and weighed on the eighth week. The mean mice weight in the GC-treated group $(19.8 \pm 1.9 \mathrm{~g})$ was significantly lower compared with the control group $(25.6 \pm 1.5 \mathrm{~g})$ following 8 weeks of treatment. The data are presented as the mean \pm standard deviation ( ${ }^{*} \mathrm{P}<0.05$, vs. the control group). GC, glucocorticoid.

head were observed after 8 weeks in the GC-treated group (Fig. 2).

Effect of GCs treatment on the expression levels of RANKL and $O P G$, as measured by immunohistochemistry. RANKL and OPG are essential for the regulation of various features of osteoclast functions, including proliferation, differentiation, fusion, activation and apoptosis (17). In particular, the balance between OPG and RANKL was demonstrated to modulate bone formation and resorption (18). Therefore, the cellular localization and protein expression of RANKL and OPG was assessed by immunohistochemistry. As demonstrated in Fig. 3, a lower positivity for OPG was observed in the control group compared with the higher OPG staining in the GC-treated group $(\mathrm{P}<0.05)$. No significant difference was indicated for the expression of RANKL between the GC-treated and the control groups ( $P>0.05$; Fig. 4). These results indicated that the greater expression of OPG inhibited osteoclastogenesis following dexamethasone and asparaginase treatment.

GC treatment inhibits osteoclast differentiation. To confirm the effect of GC treatment on the number of osteoclasts, the femoral head sections were stained with TRAP. Only TRAP-positive multinucleated cells located at the bone surface within the bone destruction were considered to be osteoclasts. Compared with the control group, the number of osteoclasts in the areas of bone destruction was significantly decreased in the GC-treated group $(\mathrm{P}<0.05$; Fig. 5). These results indicated that $\mathrm{GC}$ treatment inhibited osteoclast differentiation and function.

GCs treatment increases the mRNA expression levels of osterix and osteocalcin, and decreases the expression of MMP-9 to regulate the differentiation and function of osteoblasts and osteoclasts. In order to identify the in-depth mechanism by which GCs regulate osteoclast differentiation, the mRNA expression levels of Runx2, osterix, osteocalcin and MMP-9 were determined. Runx2 is essential for osteoblastic differentiation and skeletal morphogenesis, and acts as a scaffold for nucleic acids and regulatory factors involved in skeletal gene expression. Osterix is a zinc finger-containing transcription factor expressed in osteoblasts that serves a role in the osteoblast 

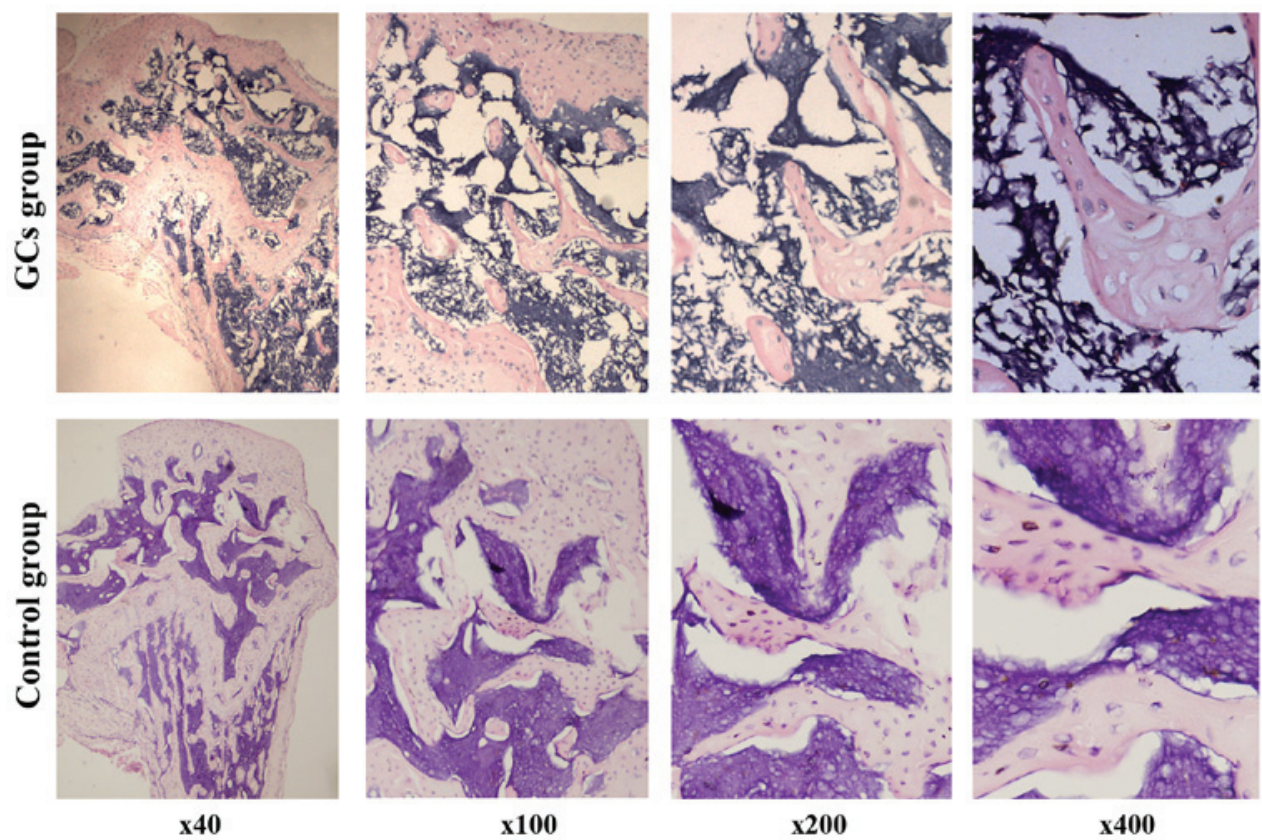

Figure 2. Effect of GCs on the histological morphology of bone formation. Histological changes of femur specimens were observed by hematoxylin and eosin staining under a light microscope. In the GC-treated group, an empty lacunae within the necrotic bone trabeculae, bone marrow cells, including adipocytic necrosis and an accumulation of cell debris in the medullary space in most areas of the femoral head, were observed in 16/37 (43\%) mice. No lesions were observed in the control group. GC, glucocorticoid.
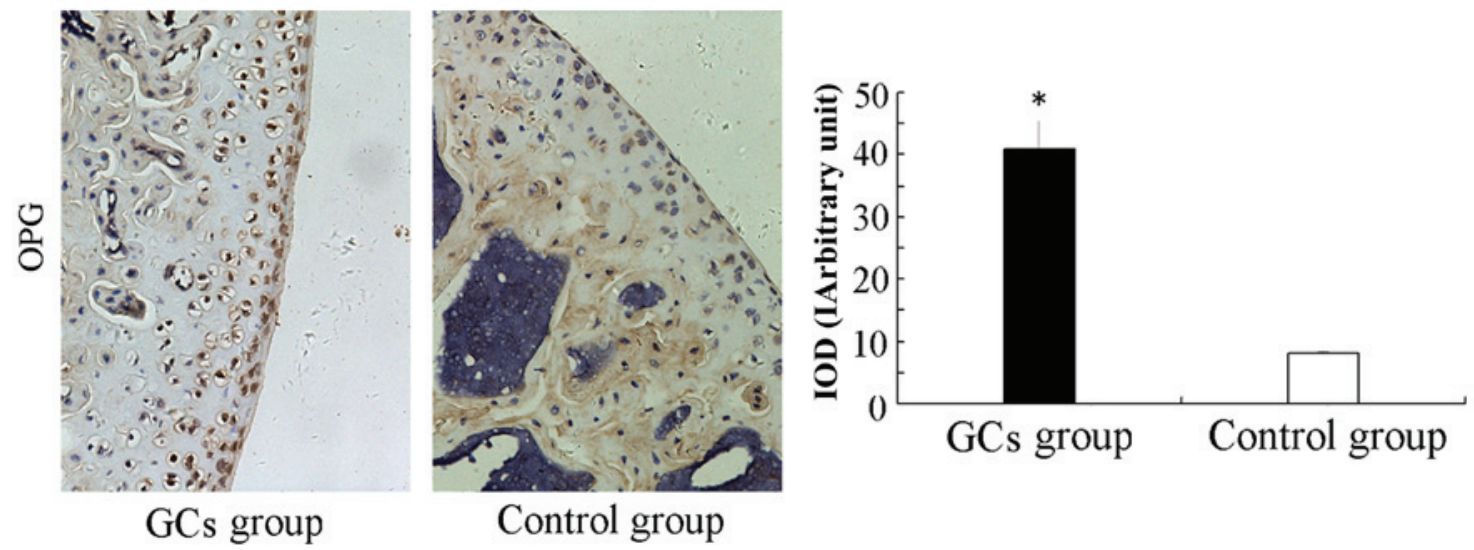

Figure 3. Effect of GC treatment on the expression of OPG. Cellular localization and protein expression of OPG were measured using immunohistochemistry. A lower expression of OPG was observed in the control group. The data are presented as the mean \pm standard deviation ("P<0.05, vs. the control group). GC, glucocorticoid; OPG; osteoprotegerin; intracellular optic density value of immunohistochemical staining.

differentiation pathway. It was suggested that Runx2 acts upstream of osterix, as previous studies demonstrated that in Runx2-null mice, osterix is not expressed, whereas in osterix null mice, Runx2 is expressed (19). As demonstrated in Fig. 6, the relative mRNA expression of Runx2 in the GC-treated group remained unchanged compared with the control group. In addition, the relative mRNA expression of osterix was increased in the GC-treated group compared with the control group ( $\mathrm{P}<0.05$; Fig. 6$)$. The results indicated that GC treatment promoted osteoblast differentiation in the late stage, however had no effect on osteoblast differentiation in the early stage.

Furthermore, osteocalcin is secreted solely by osteoblasts, is pro-osteoblastic, or bone-building, by nature, and may serve a role in the body's metabolic regulation (20). In addition, it is implicated in bone mineralization and calcium ion homeostasis
(21). MMP belongs to the zinc-binding endopeptidase enzyme family and is essential for extracellular matrix degradation in a variety of organs, including bones. In particular, high expression of MMP-9 by osteoclasts has an important role in the degradation of the extracellular matrix. As demonstrated in Fig. 6, the GC-treated group exhibited a higher level of osteocalcin and a lower level of MMP-9 compared with the control group $(\mathrm{P}<0.05)$. The results demonstrated that $\mathrm{GC}$ treatment has an inhibitory effect on osteoclast differentiation and function.

\section{Discussion}

The pathophysiology of non-traumatic osteonecrosis is more complex compared with traumatic osteonecrosis. As a common cause of non-traumatic osteonecrosis, GCs affect various steps of 


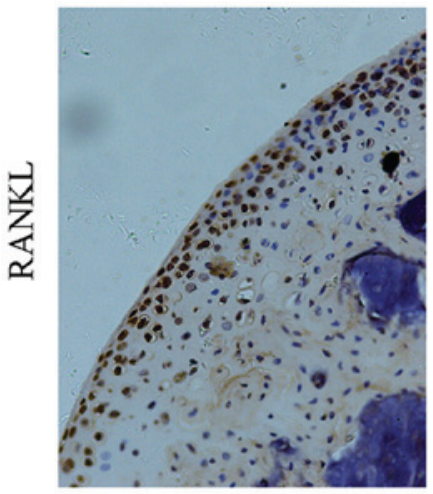

GCs group

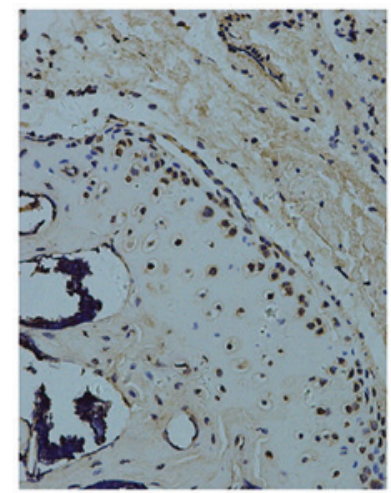

Control group

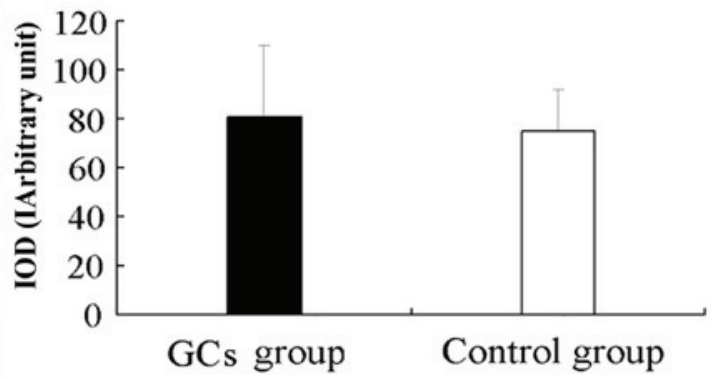

Figure 4. Effect of GCs treatment on the expression of RANKL. Cellular localization and protein expression of RANKL were measured using immunohistochemistry. No significant difference in RANKL expression was observed between the GC-treated and control groups. The data are presented as the mean \pm standard deviation. GC, glucocorticoid; RNAKL, receptor activator of nuclear factor- $\kappa \mathrm{B}$ ligand; intracellular optic density value of immunohistochemical staining.

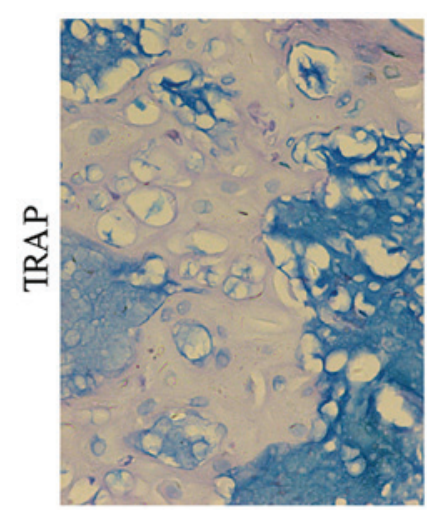

GCs group

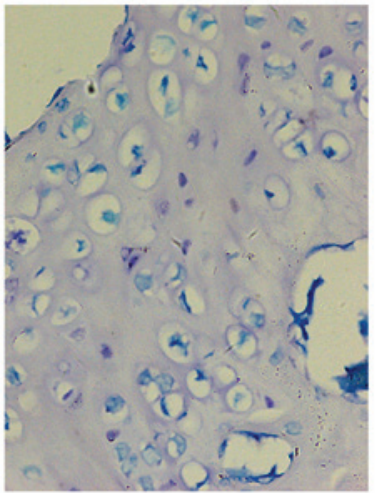

Control group

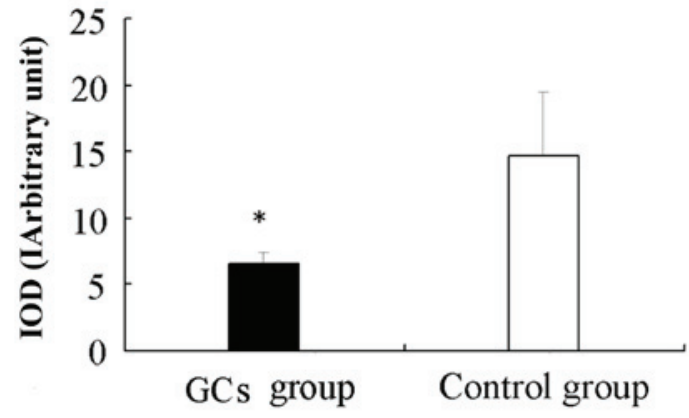

Figure 5. Effect of GCs treatment on osteoclast differentiation, as measured by TRAP staining. TRAP-positive multinucleated cells located at the bone surface within the bone destruction were considered to be osteoclasts. Compared with the control group, the number of osteoclasts in the areas of bone destruction was significantly decreased in the GC-treated group. The data are presented as the mean \pm standard deviation (*P<0.05, vs. the control group). GC, glucocorticoid; TRAP, tartarate-resistant acid-phosphatase; intracellular optic density value of immunohistochemical staining.

A

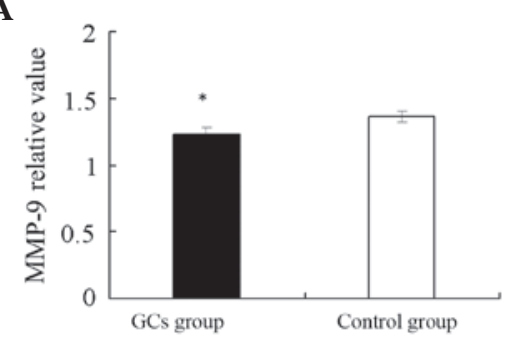

C

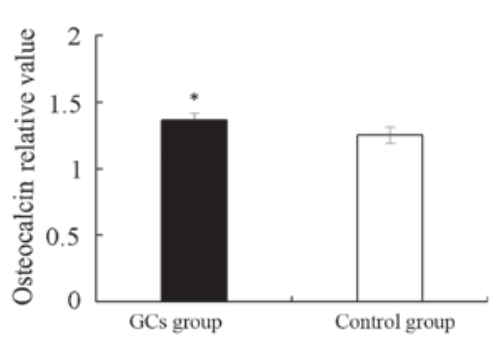

B

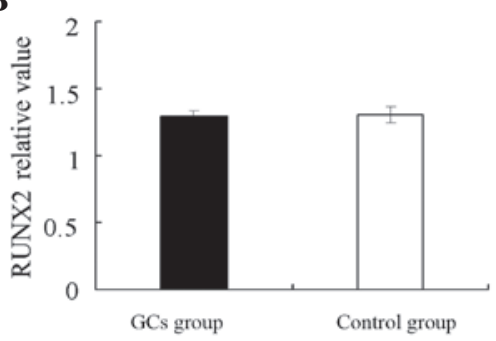

D

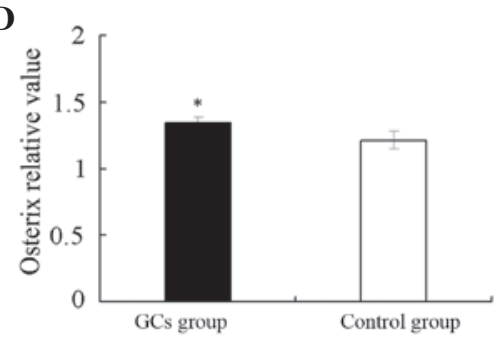

Figure 6. Effect of GC treatment on the mRNA expression of Runx2, osterix, osteocalcin and MMP-9, as measured by quantitative polymerase chain reaction. The mRNA expression levels of (A) MMP-9 increased, (B) of Runx 2 remained the same and the expression levels of (C) osteocalcin and (D) osterix increased in the GC-treated group. The data are presented as the mean \pm standard deviation ( $\mathrm{P}<0.05$, vs. the control group). MMP, matrix metallopeptidase; GC, glucocorticoid; Runx2, runt-related transcription factor 2. 
bone formation, including osteoclast or osteoblast differentiation, lipid metabolism and expression of associated transcription factors. Almost all bone diseases, including osteonecrosis, are associated with the imbalance of bone remodeling. Bone remodeling is primarily maintained by the dynamic balance of osteoclast and osteoblast activity. Osteoblasts synthesize the new bone and osteoclasts digest the old one. Osteoblasts and osteoclasts interact with each other to complete their functions.

Osteoblasts, as specialized bone-forming cells, have a variety of roles in bone remodeling, including the expression of osteoclastogenic factors, synthesis of bone matrix and bone mineralization. Pluripotent mesenchymal precursor cells differentiate into a variety of cells, including adipocytes, myocytes, chondrocytes and osteoblasts, under the guidance of a series of regulatory transcription factors, including Runx2 and osterix (22).

The expression of Runx 2 occurs prior to the differentiation of osteoblasts and only in mesenchymal precursor cells destined to chondrocytes or osteoblasts. Runx 2 is considered to be the predominant transcription factor that regulates bone formation $(23,24)$, since Runx2-knockout mice lack cartilage and osteoblasts $(25,26)$. Runx 2 is also essential during the process of expression of osteocalcin, a specific marker of late osteoblasts (27). Through the regulation of osteocalcin expression, Runx 2 promotes bone formation by differentiated osteoblasts.

Osterix is a transcription factor acting downstream of Runx2 during osteoblast differentiation $(19,26)$. Osterix knockout mice develop normally in the shape of a skeleton composed only of cartilage, and exhibit no osteoblasts or the mineralized matrix. This normal cartilage skeleton contains fully differentiated chondrocytes, thus it is hypothesized that osterix has a specific role downstream of Runx2 during osteoblast differentiation (28). Runx 2 acts at the initial stage of the differentiation of the mesenchymal precursor cells into preosteoblasts, and osterix predominantly acts at a later stage to promote the process of differentiation of the preosteoblast into the functional osteoblast (19). The two transcriptional factors, Runx2 and osterix, regulate the process of osteoblast differentiation.

Thus far, the influence of GCs on osteoblast and associated transcription factors is controversial. A previous study demonstrated that elevated Runx 2 levels in osteonecrotic cases resulted in an increase of osteoblasts, correspondingly (29). Weinstein et al (30) demonstrated that GC-induced osteonecrosis resulted in decreased osteoblasts in vivo. Additional studies indicated that the osteogenic abilities of bone marrow stromal cells are not defective in osteonecrotic cases $(31,32)$. The results of the present study demonstrated unchanged mRNA levels of Runx 2 and increased levels of osterix in the GC-treated group, compared with the control group. Therefore, the data indicated that GCs promoted the differentiation of pre-osteoblast into functional osteoblast, but cannot influence the stage of the differentiation of mesenchymal precursor cells into pre-osteoblasts in the GC-induced osteonecrosis mice model.

Osteoclasts, derived from hematopoietic stem cells, have a key role in bone resorption in bone remodeling (33). Osteoclasts differentiation and function are determined by the RANKL/OPG ratio. OPG is a cognate inhibitor of RANKL and a physiologically negative regulator of osteoclastogenesis (34-36). Previous studies indicated that only undifferentiated cells assist osteoclastogenesis, compared with fully or partially differentiated cells (36). The overall number of RANKL and OPG depends on the differentiation stage of osteoblasts. Pre-osteoblast cells express low levels of OPG and relatively high levels of RANKL, resulting in enhancement of the osteoclast differentiation and function. However, mature osteoblasts express low levels of RANKL and relatively high levels of OPG, resulting in reduction of osteoclast differentiation and function.

The results of the present study demonstrated a significant decrease in the RANKL/OPG ratio and TRAP-positive multinucleated cells in the GCs-induced osteonecrosis mouse model, indicating that the osteoclast number was decreased, and the osteoclast activity was inhibited. According to the hypothesis that expression of RANKL and OPG by osteoblasts depends on the differentiation stage of the osteoblasts, the present study hypothesized that due to overexpression of osterix induced by GCs, mature osteoblasts were increased, resulting in an increase of OPG. The RANKL/OPG ratio was subsequently decreased and osteoclast activity was inhibited. The results of the present study demonstrated that an increase in the number of mature osteoblasts may led to overexpression of osteocalcin, an inhibitor of osteoclasts. Osteoblasts and other cells, such as fibroblasts, have two specific transcripts, one encoding Runx 2 and the other osteocalcin, and the transcripts are expressed upon differentiation of these cells. Furthermore, the results indicated that the mRNA expression of MMP-9 was reduced in the GC-treated group compared with the control group. It is widely accepted that MMP-9, primarily expressed by destined osteoclast precursors and osteoclasts, has an important role in bone resorption and formation (37). Therefore, the present study hypothesized that GCs may further inhibit osteoclast activity by reducing the mRNA expression of MMP-9.

In conclusion, the present study demonstrated that in the osteonecrosis mouse model, GCs decreased osteoclast differentiation by decreasing the RANKL/OPG ratio, and additionally inhibited osteoclast function by decreasing TRAP-positive multinucleated cells and MMP-9 expression. The data of the present study suggested that the GCs decreased formation/differentiation of osteoclasts, therefore, reducing the ability of osteoclasts to resorb the 'old bone', resulting in bone necrosis.

\section{Acknowledgements}

The present study was supported by the National Natural Science Foundation of China (no. 81070688).

\section{References}

1. DiGiovanni CW, Patel A, Calfee R and Nickisch F: Osteonecrosis in the foot. J Am Acad Orthop Surg 15: 208-217, 2007.

2. Lafforgue P: Pathophysiology and natural history of avascular necrosis of bone. Joint Bone Spine 73: 500-507, 2006.

3. Powell C, Chang C and Gershwin ME: Current concepts on the pathogenesis and natural history of steroid-induced osteonecrosis. Clin Rev Allergy Immunol 41: 102-113, 2011.

4. Chao YC, Wang SJ, Chu HC, Chang WK and Hsieh TY: Investigation of alcohol metabolizing enzyme genes in Chinese alcoholics with avascular necrosis of hip joint, pancreatitis and cirrhosis of the liver. Alcohol 38: 431-436, 2003. 
5. Juéry P: Avascular necrosis after a steroid injection. CMAJ 176: 814, author reply 814, 2007.

6. Baksi DP: Treatment of post-traumatic avascular necrosis of the femoral head by multiple drilling and muscle-pedicle bone grafting. Preliminary report. J Bone Joint Surg Br 65: 268-273, 1983.

7. Lee CK and Hansen HR: Post-traumatic avascular necrosis of the humeral head in displaced proximal humeral fractures. J Trauma 21: 788-791, 1981.

8. Laroche M: Intraosseous circulation from physiology to disease. Joint Bone Spine 69: 262-269, 2002.

9. Dannemann C, Grätz KW, Riener MO and Zwahlen RA: Jaw osteonecrosis related to bisphosphonate therapy: A severe secondary disorder. Bone 40: 828-834, 2007.

10. Martí-Carvajal A, Dunlop R and Agreda-Perez L: Treatment for avascular necrosis of bone in people with sickle cell disease. Cochrane Database Syst Rev (4): CD004344, 2004.

11. Levasseur R: Mechanisms of osteonecrosis. Joint Bone Spine 75: 639-642, 2008.

12. Yamamoto T, Hirano K, Tsutsui H, Sugioka Y and Sueishi K: Corticosteroid enhances the experimental induction of osteonecrosis in rabbits with Shwartzman reaction. Clin Orthop Relat Res (316): 235-243, 1995.

13. Yamamoto T, Irisa T, Sugioka Y, Sueishi K, Yamamoto T, Irisa T, Sugioka Y and Sueishi K: Effects of pulse methylprednisolone on bone and marrow tissues: Corticosteroid-induced osteonecrosis in rabbits. Arthritis Rheum 40: 2055-2064, 1997.

14. Kabata T, Kubo T, Matsumoto T, Hirata T, Fujioka M, Takahashi KA, Yagishita S, Kobayashi M and Tomita K: Onset of steroid-induced osteonecrosis in rabbits and its relationship to hyperlipaemia and increased free fatty acids. Rheumatology 44 1233-1237, 2005.

15. Yoneda T, Williams $\mathrm{P}$, Rhine C, Boyce BF, Dunstan C and Mundy GR: Suramin suppresses hypercalcemia and osteoclastic bone resorption in nude mice bearing a human squamous cancer. Cancer Res 55: 1989-1993, 1995.

16. Livak KJ and Schmittgen TD: Analysis of relative gene expression data using real-time quantitative PCR and the 2(-Delta Delta C(T)) Method. Methods 25: 402-408, 2001

17. Hofbauer LC, Neubauer A and Heufelder AE: Receptor activator of nuclear factor-kappa B ligand and osteoprotegerin: Potential implications for the pathogenesis and treatment of malignant bone diseases. Cancer 92: 460-470, 2001.

18. Blair JM,Zheng Y and Dunstan CR: RANK ligand. Int J Biochem Cell Biol 39: 1077-1081, 2007.

19. Nakashima K, Zhou X, Kunkel G, Zhang Z, Deng JM, Behringer RR and de Crombrugghe $B$ : The novel zinc finger-containing transcription factor osterix is required for osteoblast differentiation and bone formation. Cell 108: 17-29, 2002.

20. Lee NK, Sowa H, Hinoi E, Ferron M, Ahn JD, Confavreux C, Dacquin R, Mee PJ, McKee MD, Jung DY, et al: Endocrine regulation of energy metabolism by the skeleton. Cell 130 456-469, 2007.

21. Zoch ML, Clemens TL and Riddle RC: New insights into the biology of osteocalcin. Bone 82: 42-49, 2016.
22. Galindo M, Pratap J, Young DW, Hovhannisyan H, Im HJ, Choi JY, Lian JB, Stein JL, Stein GS and van Wijnen AJ: The bone-specific expression of Runx2 oscillates during the cell cycle to support a G1-related antiproliferative function in osteoblasts. J Biol Chem 280: 20274-20285, 2005.

23. Lian JB and Stein GS: Runx2/Cbfa1: A multifunctional regulator of bone formation. Curr Pharm Des 9: 2677-2685, 2003.

24. Karsenty G: Role of Cbfa1 in osteoblast differentiation and function. Semin Cell Dev Biol 11: 343-346, 2000.

25. Ducy P, Zhang R, Geoffroy V, Ridall AL and Karsenty G: Osf2/Cbfa1: A transcriptional activator of osteoblast differentiation. Cell 89: 747-754, 1997.

26. Komori T, Yagi H, Nomura S, Yamaguchi A, Sasaki K, Deguchi K, Shimizu Y, Bronson RT, Gao YH, Inada M, et al: Targeted disruption of Cbfa1 results in a complete lack of bone formation owing to maturational arrest of osteoblasts. Cell 89: 755-764, 1997.

27. Mackie EJ: Osteoblasts: Novel roles in orchestration of skeletal architecture. Int J Biochem Cell Biol 35: 1301-1305, 2003.

28. Harada S and Rodan GA: Control of osteoblast function and regulation of bone mass. Nature 423: 349-355, 2003

29. Tingart M, Beckmann J, Opolka A, Matsuura M, Wiech O, Grifka J and Grässel S: Influence of factors regulating bone formation and remodeling on bone quality in osteonecrosis of the femoral head. Calcif Tissue Int 82: 300-308, 2008.

30. Weinstein RS, Jilka RL, Parfitt AM and Manolagas SC: Inhibition of osteoblastogenesis and promotion of apoptosis of osteoblasts and osteocytes by glucocorticoids. Potential mechanisms of their deleterious effects on bone. J Clin Invest 102: 274-282, 1998.

31. Wyles CC, Houdek MT, Crespo-Diaz RJ, Norambuena GA, Stalboerger PG, Terzic A, Behfar A and Sierra RJ: Adipose-derived mesenchymal stem cells are phenotypically superior for regeneration in the setting of osteonecrosis of the femoral head. Clin Orthop Relat Res 473(10): 3080-3090, 2015.

32. Gao YS and Zhang CQ: Comment on: Osteogenic abilities of bone marrow stromal cells are not defective in patients with osteonecrosis. Int Orthop 34: 457, author reply 459, 2010.

33. Teitelbaum SL: Bone resorption by osteoclasts. Science 289: 1504-1508, 2000.

34. Theoleyre S, Wittrant Y, Tat SK, Fortun Y, Redini F and Heymann D: The molecular triad OPG/RANK/RANKL: Involvement in the orchestration of pathophysiological bone remodeling. Cytokine Growth Factor Rev 15: 457-475, 2004.

35. Yamada Y, Ando F, Niino N and Shimokata H: Association of polymorphisms of the osteoprotegerin gene with bone mineral density in Japanese women but not men. Mol Genet Metab 80: 344-349, 2003.

36. Gori F, Hofbauer LC, Dunstan CR, Spelsberg TC, Khosla S and Riggs BL: The expression of osteoprotegerin and RANK ligand and the support of osteoclast formation by stromal-osteoblast lineage cells is developmentally regulated. Endocrinology 141: 4768-4776, 2000.

37. Grässel S, Beckmann J, Rath B, Vogel M, Grifka J and Tingart M: Expression profile of matrix metalloproteinase-2 and -9 and their endogenous tissue inhibitors in osteonecrotic femoral heads. Int J Mol Med 26: 127-133, 2010. 